\title{
Il problema della traduzione e il "linguaggio delleeineffabile" nella Commedia di Dante in rapporto alla traduzione russa del poema
}

\author{
Kristina LANDA \\ Università di Bologna \\ Italia
}

Riassunto: Il presente articolo tratta del -linguaggio dell'ineffabilitàll di Dante Alighieri alla luce della teoria della traduzione, e di alcune strutture di questo linguaggio nella traduzione russa della Divina Commedia di M.L. Lozinskij. Molti hanno scritto sul linguaggio di Dante come mezzo d'espressione delle verità mistiche. Eppure non esiste un lavoro critico in cui questo tema si studi dal punto di vista della scienza traduttiva. Il nostro obiettivo è dimostrare l'importanza del tema della -traduzionell delle verità divine nel linguaggio umano per la poetica dantesca e scoprire come tale -traduzionell si effettui nella traduzione russa della Commedia ad alcuni livelli testuali.

Parole-chiave: linguaggio dell'ineffabilità dantesco, metafora dell'immagine e del sigillo, dottrina dell'immagine e della somiglianza, creazione dantesca come traduzione, traduzione di Lozinskij, linguaggio dell'ineffabile nella traduzione

\begin{abstract}
The present paper depicts Dante's -language of the ineffablell in light of Translation Theory and of some structures of this language present in M. L. Lozinskij's Russian translation of the Divine Comedy. Much has been written about Dante's use of language as a way to express mystical truths, but not from a Translation Studies perspective. Therefore our aim is to prove the importance of -translating\| the divine truths in human language for Dante's poetics and discover how that kind of -translation\| is being recreated in the Russian version of the Comedy at a textual level.
\end{abstract}

Key words: Dante's -language of the ineffablell, the metaphors of the image and of the seal, the doctrine of the image and of similarity, the Dantesque creation as translation, Lozinskij's translation, language of the ineffable in translation.

Lo scopo del presente articolo è mettere in luce alcuni aspetti della poetica della Divina Commedia in rapporto al concetto di traduzione e al problema specifico della traduzione del poema in russo. Siccome il tema è ampio e potrebbe riguardare diversi campi dello scibile umano, in questo studio mi concentrerò solo su singoli aspetti di questa problematica, e soprattutto su un brano del poema che, a mio 
parere, potrebbe illustrare dal punto di vista metaforico il concetto stesso di traduzione e descrivere il legame metafisico fra questo concetto e il concetto dell'universo nell'epoca dantesca. Questo legame, poi, è imperniato sul problema del linguaggio dell'ineffabilità, ovvero dell'ineffabile, in Dante ${ }^{41}$, che è una delle peculiarità più importanti che caratterizzano il suo poema. Sempre nell'ambito della traduzione, è mia intenzione proporre una ricostruzione di alcuni aspetti del linguaggio dellineffabile dantesco nella sua variante tradotta in russo. A tal fine presenterò una breve analisi di una terzina del poema, appartenente al campo tematico del linguaggio dellineffabile, confrontandola con la sua traduzione russa più famosa. Quest'analisi, però, rappresenta una delle tappe iniziali di una ricerca più ampia che mira a scoprire e a ricostruire alcune strutture del linguaggio dell'ineffabile nelle traduzioni russe della Commedia. Nell'ambito di questa futura ricerca sarà definito con più precisione il campo stesso del linguaggio dell'ineffabile nella Commedia, e saranno studiati i brani del prototesto e del metatesto, significativi dal punto di vista dell'ineffabilità dantesca.

Il problema della traducibilità e della traduzione, nel discorso su Dante, risale a Dante stesso. Infatti, come possiamo leggere nel suo Convivio, l'autore, che deve difendere la sua decisione di comporre le canzoni e il commento ad esse in lingua volgare, affronta quest'argomento fin dai primi capitoli:

"E però sappia ciascuno che nulla cosa per legame musaico armonizzata si può de la sua loquela in altra transmutare, sanza rompere tutta sua dolcezza e armonia. E questa è la cagione per che Omero non si mutò di greco in latino come l'altre scritture che avemo da loro. E questa è la cagione per che li versi del Salterio sono sanza dolcezza di musica e d'armonia; ché essi furono transmutati d'ebreo in greco e di greco in latino, e ne la prima transmutazione tutta quella dolcezza venne meno." (Convivio I vii 14-16).

In altre parole, egli afferma categoricamente che una buona traduzione poetica non è realizzabile e che, anzi, tale traduzione (a differenza di quella della prosa) potrebbe risultare soltanto un atto distruttivo nei confronti della "dolcezza ed armonia" della poesia originale, la quale, per Dante, "non è altro che una composizione ad arte fatta di retorica e di musica"42 (De Vulgari Eloquentia, II, iv, 2). Eppure, come dimostra Massimiliano Chiamenti nel suo ricchissimo studio Dante Alighieri traduttore, "critica e poesia" non solo "spesso

${ }^{41}$ Il termine fu coniato da M. Colombo nel suo libro Dai mistici al Dante: il linguaggio dell'ineffabilità (1987); lo uso qui agli scopi della mia ricerca, non precisamente nello stesso significato che gli attribuisce la studiosa, ma definendo, con questo termine, tutta la parte del poema in cui l'autore trasmette la sua esperienza mistica, anche e soprattutto attraverso il riconoscimento dell'inadeguatezza del proprio linguaggio poetico per tale trasmissione.

42 "Nichil aliud est quam fictio rethorica musicaque poita" (2011, trad. di M. Tavoni, curatore dell'edizione dalla quale si cita). 
vanno insieme" (1995, 195), ma talvolta possono anche contraddirsi: così, nello stesso Dante, nonostante la rigidità della tesi riportata sopra, si ritrovano alcuni brillanti esempi di traduzioni fatte da lui. Come esempio più palese Chiamenti indica i versi più significativi per Dante $\mathrm{e}$ per tutta la cultura del suo tempo, come quelli virgiliani delle Bucolicae IV, 5-7, volgarizzati da Dante nel XXII canto del Purgatorio (Purgatorio, XXII, 55-72, cit. in Chiamenti 205). Inoltre, come risulta dalla sua ricerca, l'intero testo della Commedia è intriso di traduzioni, che Chiamenti suddivide in diverse tipologie, dai "trasferimenti diretti" e "modulazioni" degli autori latini, fino alle "autotraduzioni", cioè i brani di alcune opere volgari di Dante da lui stesso tradotte in latino (1995, 11-194). Certamente, non c'è da meravigliarsi se l'Alighieri, nelle vesti del poeta, del filosofo e del teologo, avendo a che fare con una civiltà contrassegnata da due tradizioni linguistiche (quella latina, per i chierici e gli studiosi, e quella volgare, per il resto d'Italia; la prima usata per trattati religiosi e scientifici e la seconda per la poesia d'amore), abbia dovuto affrontare il problema della traducibilità, definirlo e risolverlo con modalità alcune volte forse inaspettate per lui stesso.

Inoltre, il problema della traducibilità non si pone, per Dante, solo sul piano esteriore, of fisico, trattato nel libro di Chiamenti, a livello cioè delle citazioni palesi e nascoste, dell'intertesto e delle reminiscenze, della traduzione poetica, eccetera. In realtà questo tema è ben più profondo per il nostro poeta e andrebbe considerato anche a livello metafisico, ovvero a livello di compenetrazione fra la realtà trascendente e il tessuto verbale. A questo livello è stato definito più volte come problema dell'ineffabilità da alcuni studiosi come la già citata M. Colombo, M. Ariani (2010, 277-289), M. Mocan (2007, 147177) e molti altri. Questo problema, che consiste nella difficoltà di trasmettere le realtà divine con le parole umane, viene affrontato da Dante a partire dai canti iniziali del Purgatorio, ma soprattutto nelle ultime pagine del poema, per mezzo delle metafore della luce, del fuoco e della visione. Questo spinge inevitabilmente a riflettere su un tema che da sempre accompagna la riflessione sulla traduzione, cioè se sia possibile trasferire un messaggio in un sistema semiotico diverso da quello originale ${ }^{43}$. Ciò di cui si convince un lettore attento a queste problematiche dopo aver letto le ultime terzine del XXXIII del Paradiso, è che Dante, oltre ad essere poeta, teologo, filosofo, maestro e

43 Infatti, come afferma, per esempio, il teorico russo della traduzione Ju.S. Stepanov: - "perevod možno bylo by sdelat' osnovnym, neopredeljaemym ponjatiem semiotiki, a perevodimost' otoždestvljat' so smyslom" (Francuzskaja stilistika 260, cit. in Garbovskij 2004, 243). Si potrebbe rendere la "traduzione" un concetto base della semiotica, che non necessiti di definizioni, e si potrebbe identificare la "traducibilità" con il "senso". - La traduzione dal russo qui e in seguito è mia. Così il termine "traduzione" potrebbe essere usato anche per descrivere il rapporto fra la "visione" di Dante e il sistema verbale del testo della Commedia, come un concetto base per descrivere il processo del passaggio dal sistema semiotico delle realtà divine a quello del linguaggio poetico. 
discepolo dei suoi maestri, fu anche un eccellente traduttore, quasi un Giacobbe in lotta continua con Dio, che, perfettamente consapevole della propria sconfitta finale, decide comunque di lottare e che quindi, pur perdendo, paradossalmente vince.

Questa metafora di autore del testo come Giacobbe potrebbe essere perfettamente adoperata, al mio parere, anche nel caso delle traduzioni della Commedia in altre lingue, nelle quali, come nello specchio, si riflette il tentativo originale del poeta fiorentino di vincere la propria materia.

L' amorosa lotta fra il creatore e la sua creazione, nel doppio processo della composizione del poema e della sua traduzione in altre lingue, potrebbe essere interpretata nei termini platonici ed aristotelici cari a Dante, e cioè nei termini dell" "immagine" e "suggello":

La moto e la virtù d'i santi giri, come dal fabbro l'arte del martello, da' beati motor convien che spiri;

e 'l ciel cui tanti lumi fanno bello, de la mente profonda che lui volve prende l'image e fassene suggello44. (Paradiso, II, 127-132)

Parafrasato, il brano significa questo:

E' necessario (conven) che il movimento e la virtù dei cieli santi dipendano da beati enti motori (cioè dalle intelligenze angeliche), come l'arte del martello dipende dal fabbro... e il cielo che si adorna di tante stelle (l'ottavo) riceve dalla mente profonda, o intelligenza angelica, che lo muove la forma esemplare (l'image) di cui si fa sigillo (per improntarne la materia terrestre). (Chiavacci Leonardi 2001, 41-42)

Senza inoltrarci adesso nella storia di questa metafora nel pensiero europeo, a partire dai filosofi greci fino a Dante - per uno studio approfondito dell'argomento rinvio il lettore, per esempio, al saggio di G. Stabile "Teoria della visione come teoria della conoscenza" (2007, 9-29) - ce ne possiamo servire invece per provare a raffigurare simbolicamente il processo della traduzione di Dante in un'altra lingua. Infatti, potremmo intendere le intelligenze angeliche come realtà ineffabili, ovvero trascendentali, che Dante cerca di trasmettere al suo lettore; il cielo stellato come materia (linguaggio della poesia dantesca) e la forma esemplare ("l'image") come messaggio che si riesce a trasmettere in questa materia. E' facile, poi, notare che il processo della trasmissione, o della comunicazione, non si ferma sul cielo stellato: nella metafora, infatti, si crea l'effetto di un doppio sigillo, e il cielo, divenuto esso stesso un unico soggetto formante, comunica la forma al mondo terrestre. Così, potremmo intendere il cielo formato dagli angeli

44 Per la mia ricerca uso il testo della Commedia nella versione di G. Petrocchi, commentato da A.M. Chiavacci Leonardi ricorrendo, in caso di necessità, ad altri commenti. 
come il testo della Commedia di Dante, la terra che riceve la forma di questo cielo come materiale di un'altra lingua, e la forma ricevuta dalla terra da parte del cielo come messaggio poetico, riprodotto in quest'altro materiale.

Lo schema del processo descritto da Dante in linea con il modello aristotelico, in pratica, sarebbe questo:

intelligenze angeliche-comunicazione della forma al materiale $>$ cielo stellato-comunicazione della forma al materiale -> terra.

Lo schema dei due processi (della composizione della Commedia e della sua traduzione in un'altra lingua), metaforicamente paragonato al primo schema, sarebbe il seguente:

realtà ineffabili-trasmissione del messaggio trascendentale al linguaggio dantesco $\rightarrow$ testo della Commedia-trasmissione del messaggio poetico al linguaggio dei traduttori -> testo delle traduzioni.

Questo paragone metaforico è suggerito da alcune ragioni, sia di carattere filosofico e letterario che traduttologico e semiotico. In primo luogo, come è stato indicato sopra, è Dante stesso a presentare la sua opera come tentativo di traduzione (nel senso semiotico del termine) dal linguaggio divino al linguaggio umano (vedi, per esempio, Paradiso XXX 31-33, 97-99, XXXIII 55-57, 70-72, 121-123 ecc.). Al contempo, ispirato all'idea platonico-aristotelica del sigillo, che trasmette la forma alla materia, e alla dottrina cristiana dell'immagine e della somiglianza delle creature a Dio45, egli disegna l'universo del poema come un mondo plasmato secondo il modello divino. L'immagine inalterata di Cristo custodita nel cuore delle persone, di cui parla San Bernardo ${ }^{46}$, scelto da Dante per il ruolo di sua ultima guida, è una specie di "invariante" che riunisce i due mondi: il cielo e la terra, e che permette all'uomo di riconoscere la propria origine: Dio. La metafora del sigillo che plasma il materiale pronto a ricevere la forma dal soggetto formante diventa, in questo senso, metafora chiave per descrivere il processo della creazione e il meccanismo stesso che garantisce il funzionamento regolare dell'universo. Ma nel mondo dantesco esistono due creatori: Dio creatore dell'universo e il poeta creatore del testo. Il poeta protagonista, producendo il tessuto testuale del poema, si riferisce alla volontà divina che lo ispira a farlo (Paradiso XVII 127142): e sarà forse lecito supporre che, in un testo logico, coerente e matematicamente strutturato (oltre che attingente alle fonti dottrinali e alla simbologia d'uso degli autori antichi e medievali) come quello della Commedia, allo strumento di lavoro del Creatore Dio possa corrispondere, a livello simbolico, lo strumento di lavoro del creatore

$45 \mathrm{Su}$ questa dottrina dei teologi cui poteva ispirarsi Dante vedi il lavoro di R. Javelet (1967) e di Zambon (2007, 17-33).

46 A proposito della dottrina di San Bernardo di Chiaravalle vedi soprattutto la prefazione di Gilson all'edizione dei testi del mistico (1949, 8-29). 
poeta, e la metafora che descrive il meccanismo di funzionamento dell'universo reale possa descrivere anche quello dell'universo poetico.

Il legame fra Dio creatore del mondo e poeta creatore del testo fu intuito e genialmente trasferito al campo della traduzione dal poeta Osip Mandel'štam, il quale, nel suo famoso saggio Conversazione su Dante, interpreta il processo della creazione dantesca proprio come processo traduttivo:

"Il segreto della sua capienza sta nel fatto che lui non aggiunge nemmeno una parola da parte sua. E' mosso da qualsiasi cosa, tranne l'invenzione. Dante e fantasia: ma sono cose incompatibili!.. Quale fantasia avrebbe? Lui scrive sotto dettatura, è un copista, è un traduttore..." 47 .

Tracciare un paragone tra la creazione del mondo dal punto di vista dantesco ed il processo della traduzione è possibile perché entrambi i fenomeni sono accomunati dallo stesso fattore, che li condiziona nella maniera più assoluta: partono tutti e due da un originale, senza il quale, come senza l'impronta del sigillo, non potrebbe esistere né quel mondo, né un testo tradotto. Infatti, come risulta dall'analisi testuale del poema e dagli studi critici, il mondo dantesco, secondo la logica aristotelica e la visione neoplatonica ${ }^{48}$, è imperniato su un processo di comunicazione: c'è una forma ("virtù", come la definisce Dante nella metafora del sigillo) che si trasmette dall'Essere più alto dell'universo, cioè Dio, ai gradi più infimi del creato, seguendo l'ordine gerarchico, e il narratore stesso, inserendosi dentro questa struttura, diventa solo un medium fra il mondo della realtà divina e il mondo terreno della somiglianza: uno che riceve e trasmette ad altri il messaggio di virtù ricevuto. Ma se le intelligenze angeliche, trasmettendo la virtù divina alle creature inferiori, garantiscono l'esistenza stessa dell'universo, il narratore, trasmettendo il messaggio trascendentale, o ineffabile, ai suoi lettori, cerca di "rimuovere i viventi in questa vita dallo stato della miseria e condurli allo stato della felicità"49. (Epistole, XIII, 15) Questa figura del ricevente-trasmettente è fondamentale anche per la teoria della traduzione: ricordiamo, per esempio, il modello classico del processo traduttivo di Anton Popovič che presuppone il doppio ruolo del traduttore: ricevente e autore del metatesto $(2006,36)$. Così, in quest'ottica del mondo dantesco, qualsiasi traduzione del poema divino altro non sarebbe che una traduzione della traduzione, che in realtà

\footnotetext{
47 "Sekret ego ëmkosti v tom, čto ni edinogo slovečka on ne privnosit ot sebja. Im dvižet vsë, čto ugodno, tol'ko ne vydumka, tol'ko ne izobretatel'stvo. Dant i fantazija - da ved' éto nesovmestimo! [...] Kakaja u nego fantazija? On pišet pod diktovku, on perepisčik, on perevodčik..."(2001, 690). Traduzione dal russo di Remo Faccani.

48 Sul tema del neoplatonismo nel poema vedi soprattutto la voce «Platonismo» nell'Enciclopedia Dantesca (Cristiani, 1970); il libro di Bruno Nardi (1967), quello di Marco Ariani già citato e molti altri.

49 In originale: "removere viventes in hac vita de statu miserie et perducere ad statum felicitatis", la traduzione dal latino è mia.
} 
costituirebbe solo un altro elemento della "catena comunicativa" di cui parla Popovič (37) e che, dentro il sistema ontologico della Commedia, diventa il motivo principale di ogni tipo di movimento e quindi della vita stessa.

Tutto ciò ci dimostra l'importanza del concetto della traduzione e della traducibilità per il mondo del poema e di conseguenza volge l'interesse del lettore verso il mondo delle traduzioni di Dante in altre lingue, in cui potremmo forse, fra tante altre cose, ritrovare ciò che persiste nelle traduzioni rimanendo dopo l'interpretazione da parte del traduttore del testo originale, e cioè l'invariante del linguaggio dell'ineffabilità, o del messaggio di virtù dantesco. "L’informazione invariante" di cui A. Lûdskanov parla come di una parte del prototesto rimasta nel metatesto (cit. in Osimo 2013, 70), potrebbe essere paragonata nel mondo dantesco alla "forma impressa nell'uomo" dal sigillo divino "che lo rende somigliante a Dio" (Zambon 2007, 17) - e non identico a Dio. Tale identità non è mai possibile, perché permane, nell'uomo che cerca la perfezione e l'avvicinamento a Dio, una differenza probabilmente simile a quella che intercorre fra il testo originale e la sua traduzione. Ma come l'uomo, nell'immaginario medievale e nel mondo dantesco, cerca la massima somiglianza con Dio, così il traduttore "tende a fare sì che la dominante del metatesto coincida il più possibile con l'invariante, ossia cerca di preservare la dominante del cambiamento" (Osimo 2013, 288).

Se il meccanismo narrativo stesso del poema è basato sul concetto della traduzione intesa come comunicazione fra il mondo celeste, il mondo terreno e il mondo verbale; se il poema, come abbiamo visto con M. Chiamenti, è intriso di traduzioni; se Dante, sia dentro il testo del poema che all' infuori di esso, affianca alla prassi della traduzione i ragionamenti teorici su quest'argomento, mostrando, in tal modo, l'importanza del tema per lui, sarebbe forse a maggior ragione interessante tentare una ricerca dei sensi più profondi della Commedia tramite le traduzioni del suo linguaggio dell'ineffabilità in altre lingue. E' nei metatesti infatti che i significati della metafora della traducibilità dovrebbero rivelarsi e realizzarsi appieno. Studiando l'invariante ineffabile come "forma" trasmessa dal "sigillo" del testo originale alla "materia" (lingua del traduttore), e le variazioni come proprietà di questa "materia", si potrebbe vedere sotto una nuova luce le strutture profonde del testo originale e scoprire il rapporto fra queste strutture e "la combinazione di variante e invariante, determinata dalla mentalità del traduttore e dalle sue considerazioni razionali, e presenti nel metatesto" (Osimo 2013, 71).

Ma, siccome il tema "Il linguaggio ineffabile della Commedia e le sue traduzioni” è piuttosto vasto, mi concentrerei, nel lavoro presente, sulle traduzioni dantesche fatte in Russia, patria di grandi opere letterarie, di una famosa teoria della traduzione e di numerose traduzioni dei classici della letteratura mondiale. E siccome la storia delle traduzioni della Commedia in Russia risale al 1791 (Golenišev- 
Kutuzov 2011, 487-488) e continua fino ai nostri giorni, in questa sede preferirei limitarmi alla traduzione del poema, considerata classica, eseguita da Michail Lozinskij (1936-1942).

La scelta cade su quest'autore per più motivi. Il primo è che appartiene alla nuova scuola sovietica della traduzione, nata all'epoca del formalismo, e ispirata all'idea dell'equivalenza del metatesto al prototesto soprattutto a livello formale: nel caso di Dante, chiaramente, si tratta del suo ritmo, metro, quantità di terzine ecc. Questa tendenza ha influenzato moltissimo la letteratura tradotta nella seconda metà del ' 900 in Russia, e si potrebbe dire che è quella più vicina alla percezione moderna del concetto della traduzione da parte dei lettori e degli studiosi russi50.

Inoltre, la traduzione di Lozinskij è diventata una sorta di "spartiacque" fra la tradizione precedente e quella posteriore delle traduzioni dantesche in Russia: insignita del premio Stalin, fu presto considerata "classica"; tutte le traduzioni preesistenti furono aspramente criticate, mentre quelle posteriori rappresentarono tentativi di superarla ${ }^{5}$.

Infine, essendo l'interesse della mia ricerca concentrato sulla trasmissione di Dante al "ricevente" di oggi, ossia al lettore attuale, è fondamentale analizzare la traduzione della Commedia solitamente letta e studiata dai russi ancora oggi.

Come esempio dell'analisi del linguaggio dellineffabile propongo il breve studio di un brano del poema che rientra appieno nel tema del linguaggio dell'ineffabilità dantesco: canto XXXIII del Paradiso, versi 46-48. L'analisi si basa sui principi sinestetici proposti da Ju.M. Lotman e da O. Mandel'štam ${ }^{2}$ e sperimentati in parte da A. A. Iljušin. Si tratta del momento del "silenzio" finale del poema, o piuttosto dell'inizio di questo silenzio, arrivato dopo la fine della preghiera di San Bernardo, quando Maria alza gli occhi verso Dio per permettere a Dante di contemplare la Trinità. In questo silenzio, Dante s'avvicina al "fine" di tutti i suoi desideri, alla fonte e origine del creato, e la terzina diventa forse uno dei tentativi più importanti di esprimere le realtà ineffabili nel poema, attraverso la descrizione dello stato del protagonista.

Cito qui l'originale:

E io ch' al fine di tutti i disii

Appropinquava, sì com'io dovea,

l'ardor del desiderio in me finii.

\footnotetext{
50 Vedi, a proposito dell'appartenenza di Lozinskij a questa tendenza generale, per esempio, Čukovskij $(2011,79,81,253)$; a proposito del peso di questa tendenza nella traduzione poetica contemporanea, per esempio, Gasparov (2006, 7-8).

51 Vedi sulla storia della traduzione di Lozinskij in Russia almeno Lozinskij S. (1987, 10-17); Achmatova (2011, 320-322); Iljušin (1973, 217-244), Belza I. (1979, 34-73) ecc.

$5^{2}$ Si tratta di un'analisi complessa delle diverse strutture del testo, da quella fonetica a quella tematica, intrapresa per individuare i vari significati di un testo (Lotman, 64, 7889; Mandel'štam 644-698). Qua, ovviamente, non è presente che un elemento di tale analisi ideale del testo.
} 
La traduzione classica di Lozinskij è:

I ja, uže predčuvsvuja predel

Vseh voždelenij, ponevole, strastno

Predel'nym ožidan'em plamenel. (Lozinskij 1999)

Parafrasando la terzina originale, una delle commentatrici contemporanee più autorevoli del poema, A.M. Chiavacci Leonardi, propone il seguente contenuto: " $E$ io che mi avvicinavo al termine ultimo di tutti i desideri (cioè Dio), portai al suo culmine in me, così come dovevo, l'ardore del desiderio”. Eppure, già in questa interpretazione, dobbiamo affrontare un problema: il verbo "finii", nella lingua italiana, può essere interpretato in diversi modi, e in questo preciso caso la commentatrice preferisce la lezione "portai al culmine", riferendosi alle citazioni di San Tommaso e di Dante stesso (dai trattati Monarchia e Convivio). Respinge, invece, menzionandola nel suo commento, l'ipotesi di "finii" come "esaurii" e "terminai", preferita da alcuni altri commentatori (XXXIII, 48). Questa divergenza già a livello dei commenti al testo dantesco nella lingua originale ci porta, prima di tentare ogni altra analisi, a consultare i commenti utilizzati dal traduttore dell'opera dantesca. Sappiamo, dalla testimonianza di suo figlio, che Lozinskij ebbe la possibilità di consultare diverse edizioni commentate del poema, a partire da quella di Cristoforo Landino del 1481, fino a quelli a lui contemporanei: Casini e Barbi, Scartazzini e Vandelli (Belza 1989, 13). Inoltre, lui stesso era perfettamente consapevole della necessità di fare scelte, sia interpretative che traduttive, di cui parlava così:

Molti punti della Divina Commedia non sono chiari. Ci hanno lavorato i commentatori di tutti i paesi, discutendo fra di loro. Mi è toccato scegliere fra le loro interpretazioni. E lì dove il testo di Dante ammetteva diverse interpretazioni ho dovuto fare in modo che anche il testo russo potesse avere due o tre interpretazioni53. (16)

Ciò dimostra, senza dubbio, la serietà del suo approccio scientifico nel lavoro traduttivo e la totale consapevolezza della propria responsabilità di fronte ad un testo così complesso. Tornando alla terzina, se vediamo, per esempio, il commento di Scartazzini e Vandelli, l'interpretazione del passo sarà simile a quella fatta da A.M. Chiavacci Leonardi:

L'ardor... finii: molti intendono 'l'ardente desiderio cessò in me, essendo io oramai certo di essere soddisfatto'; ma "che questo ardore finisse, quando D. s'avvicinava a Dio, prima di contemplarlo, sarebbe in verità uno strano caso"; Pistelli, o. c., p. 19. Noi col Todesch., col Pistelli ed altri" (cfr. Bull. XII, 331) intendiamo finii nel senso di 'compiei', cioè

53 In originale: "Mnogie mesta "Božestvennoj Komedii” nejasny. Nad nimi trudilis' kommentatory vseh stran, sporja meždu soboj. Prihodilos' delat' vybor meždu ih tolkovanijami. A tam, gde tekst Dante dopuskaet raznyje ponimanija, nado bylo delat' tak, čtoby i russkij tekst mog byt' ponjat dvojako ili trojako." 
portai l'ardore del desiderio ch'era in me, al più alto grado a cui potesse arrivare, cosa necessaria nella vicinanza del fine di tutt'i disii, la quale importava assoluta certezza di vederlo tra breve: quando, tra un momento, avverrà la visione, allora si spegnerà l'ardore del desiderio (Scartazzini e Vandelli, Paradiso XXXIII 46-48).

Se guardiamo, ora, il testo della traduzione di Lozinskij, troviamo questa interpretazione poeticamente realizzata: "predel'noje ožidan'je" in russo corrisponde all' "attesa" (Dal' 2005, 34876) "massima, estrema" (Švedova 2005, 27224, Evgen'eva 2005, 53719, Ožegov i Švedova 2005, 26381). Inoltre, in Dante troviamo vicine le parole con la stessa radice, i cui campi semantici, come abbiamo visto sopra, si incrociano: "fine" e "finii", e questa figura retorica-tipica dello stile dantesco-è trasmessa dal traduttore attraverso la coppia di parole "predel"-"predel'ny"; cambia solo la parte del discorso: se in Dante, infatti, sono posti accanto "fine" e "finii", che sono sostantivo e verbo, in Lozinskij troviamo: "predel" e "predel'ny", che sono sostantivo e aggettivo.

L'altro problema è creato dalla coppia "disio"-“desiderio". Alla semantica di questi due concetti nel testo della Commedia, fondamentali per la sua intera comprensione, è dedicato uno studio del dantista L. Pertile, La punta del disio, che tratta da vicino le problematiche dell'esperienza ineffabile in rapporto alla natura umana del protagonista (2005). Lo studioso osserva che il termine "desiderio" ricorre nel poema solo una volta in ogni cantica, mentre il "disio" si ripete più spesso. Il termine "desiderio" etimologicamente è più vicino al sentimento della mancanza di qualcosa o qualcuno, invece il "disio" fa pensare al desiderio erotico (20). Eppure, precisa Pertile, la scelta paradigmatica dei termini nel poema non è dettata dal loro valore etimologico, ma dalla loro ascendenza letteraria, valore fonico e funzionalità ritmica; non per niente Dante raccomanda nel De vulgari eloquentia (II vii 5) l'uso di disio come forma propria del volgare illustre. (20)

Dunque, tutti e due termini, senza una netta distinzione semantica, possono essere associati, in Dante, al senso di mancanza, e ciò, precisa lo studioso, "diventa evidente se si considerano i termini con cui disio e derivati sono messi in rapporto sintagmatico: le metafore con cui Dante qualifica implicitamente il desiderio in termini di fame, sete, vuoto, mancanza" (20).

In Lozinskij questa coppia di parole diventa "voždelenij"“ožidan'em". "Voždelen'e", in russo, ha le connotazioni sia di passione erotica che di desiderio passionale e sensuale, di lussuria (Dal' 2006, 5501, Evgen'eva 2005, 6455, Švedova 2005, 3863); in Puškin troviamo le stesse connotazioni (Vinogradov 2105). "Ožidan'e", come abbiamo detto sopra, corrispone più all"'attesa". Abbiamo visto che queste accezioni rientrano nel campo semantico del "disio" dantesco, ma l'utilizzo di questi termini, proprio per tale motivo, riduce l'intero campo alle sfere determinate. Così, il "disio"/"desiderio" potrebbe avere 
anche altre accezioni, tranne la passione sensuale, mentre "voždelen'e", comunque, si associa, per il lettore russo, più con la lussuria che con il "disio" della tradizione amorosa del dolce stil novo e della ricerca dell'amore supremo nella Commedia. Ha carattere più neutro la parola "želan'e", con cui, infatti, generalmente si traduce la parola italiana "desiderio" e che è usata, per esempio, dal traduttore contemporaneo Marancman nella sua variante della terzina (2006, XXXIII 46-47).

Cosa viene aggiunto dal traduttore in questi tre versi, rispetto alla terzina originale? Potremmo seguire questi cambiamenti a più livelli, da quello fonetico a quello tematico e concettuale.

In Lozinskij troviamo l'alliterazione dei suoni $d$, $l, z ̌ z ̌ z d$. I suoni duri sono presenti nelle parole che riguardano sia il personaggio che il fine dei suoi desideri; trasmettono l'idea che il desiderio del personaggio debba necessariamente spingersi contro l'ultima barriera, oltre la quale non si potrebbe andare. I suoni dolci, dall'altra parte, dominano nelle parole che descrivono lo stato del personaggio, e ci immergono nella corrente del suo desiderio, sottolineando la dolcezza, la languidezza, la passionalità tipici del desiderio sensuale: "voždelen'e", che entra in conflitto con l'ultimo limite posto al desiderio umano, che è Dio.

In Dante troviamo, invece, l'allitterazione sulla 'd' e sulla ' $r$ ' nelle parole che riguardano soltanto il desiderio ma non il suo fine: essa significa, a mio parere, soprattutto la forza e l'ardore del desiderio stesso, la sua potenza che entra in contatto con la potenza divina, nella quale, poi, si dovrebbe esaurire. Invece nella parola che significa l'ultima barriera: "fine" di tutt'i disii c'è una mitezza che placa l'impeto estremo del personaggio.

Passando al livello lessicale, notiamo nel testo tradotto, anzitutto, la corrispondenza delle rime: "predel"-"plamenel”, dove il sostantivo che significa l'ultimo fine (barriera) corrisponde, a livello di consonanza, al verbo che significa l'ardore del desiderio. In Dante troviamo, al contrario, la corrispondenza rimica fra "disii"-"finii", che, a livello semantico, secondo l'interpretazione usata dallo stesso Lozinskij, come abbiamo visto sopra, esprime il rapporto fra i desideri crescenti e il desiderio portato al suo culmine.

Fra i lessemi presenti nei versi di Lozinskij notiamo, inoltre, quelli che divergono in maniera molto interessante da quelli danteschi. Tale, per esempio, è il gerundio "predčuvstvuja": mentre Dante usa il verbo "appropinquava" ("mi avvicinavo"), che trasmette l'idea di un cammino reale verso l'ultimo fine, Lozinskij, invece, lo traduce con un "presentendo", spostando l'azione oggettiva nel campo dei sentimenti, vale a dire di soggettività, o di psicologismo. Il discorso dei sensi, nel mondo dantesco, è molto complesso e andrebbe studiato a parte (si veda, per esempio, P. Boyde, 1993). Ed è ancora più difficile parlare della possibilità delle sensazioni e dei sentimenti umani a questo grado di salita dell'uomo verso Dio. In questo senso la parola "predčuvstvuja" di Lozinskij apporta, nel testo, un elemento di percezione moderna dei 
fenomeni, più concentrata sui processi dentro l'individuo che su quelli universali.

Un altro elemento diverso a livello lessicale aggiunto da Lozinskij è "ponevole", che significa "per necessità, contro la propria volontà" (Dal' 2005, 47966, Evgen'eva 2005, 51802). Risulta, dalla traduzione, che il personaggio finì in sé "l'ardor del desiderio" contro la propria volontà, il che contraddice completamente l'originale "sì com'io dovea": tutti i desideri dell'uomo, nella logica dantesca, confluiscono nella loro unica fonte in armonia con la volontà. Non potrebbe, nei termini della dottrina cristiana, la volontà umana essere contraria a tale eccesso del desiderio di raggiungere Dio a questo stadio della salita spirituale.

L'ultima parola aggiunta dal traduttore è "strastno": passionalmente, rafforzamento del verbo "plamenel". Tutto l'insieme di parole "strastno predel'nym ožidan'em plamenel" si sostituirebbe, nella traduzione, all'originale "l'ardor del desiderio in me finii". Nell'economia della terzina l'espressione di Dante appare molto compatta e quasi laconica, rispetto a quella di Lozinskij. L'“ardor" già contiene i significati della passione (vedi a proposito lo studio di Mira Mocan citato sopra, 2007, 33-56), e Dante non aggiunge nessun'altra caratteristica a questo sostantivo che occupa uno spazio così importante dentro la sua opera. Lozinskij, invece, aggiunge l'avverbio "strastno" al verbo che anche in russo contiene già i significati della passione (Evgen'eva 2005, 47439).

Passando alle divergenze grammaticali, forse il tratto più importante è quello dell'aspetto imperfettivo del verbo "plamenet'": mentre in Dante il passato remoto, significante un'azione conclusa, corrisponde armoniosamente alla semantica stessa del verbo "finire", in Lozinskij l'aspetto imperfettivo del verbo sottolinea lo stato duraturo del personaggio, e la semantica dell'ultimo limite è trasmessa dall'aggettivo "predel'nym", che si riferisce al desiderio, ovvero all' "attesa". E' al senso dell'attesa, infatti, che corrisponde l'aspetto imperfettivo del verbo usato dal traduttore, e riflette, qua, se non la logica interna della terzina dantesca, sicuramente il contesto tematico in cui si svolge (l'attesa della visione della Trinità).

Così, in base a queste poche osservazioni, che non pretendono di essere un'analisi completa del prototesto e del metatesto, ma solo l'esempio di un lavoro che andrebbe sviluppato in più direzioni, proviamo ora a disegnare ciò che nella traduzione permane del concetto dantesco e ciò che, invece, cambia.

Permane, evidentemente, il tema del personaggio il cui desiderio diventa "estremo" prima di raggiungere il fine di tutti i desideri. Permane il motivo del desiderio come "fiamma", grazie alla comunanza semantica fra "ardore" e "plamenel". Infine, permane l'effetto dell'allitterazione, usato anche da Dante. Al contempo, cambia, proprio a partire dall'allitterazione, il rapporto stesso fra il "fine" e il "desiderio". Mentre nel testo originale la tensione del desiderio, che ha 
portato il protagonista avanti lungo tutto il cammino del poema, si risolve nell' impeto finale, nella traduzione la tensione non solo aumenta, ma perdura, nella lotta del "desiderio" con il "fine", "predel" (che in russo è più vicino al "limite" come significato). Non ci deve sorprendere, perciò, l'apparizione del concetto del tutto assente in Dante, "ponevole", perché questo concetto è strettamente legato all'idea di una violenza, di una tensione, di un conflitto, che non si evince dal testo dantesco ma è presente nel testo della traduzione.

Tale quadro, tracciato in base ad una breve analisi di un solo brano del poema, ci permette già di affermare che esistono mondi interi dentro il testo della traduzione: mondi che riportano l'impronta del sigillo del testo originale, ma che divergono da esso nella stessa misura in cui il mondo diverge dall'unica fonte della creazione. In questi mondi, l'ineffabile che Dante cerca di tradurre nel suo poema riceve delle interpretazioni e degli sviluppi forse inaspettati, ma proprio per questo interessanti. L'analisi del brano ci autorizza a supporre che anche il testo della traduzione usi il proprio linguaggio dell'ineffabile, vale a dire le strutture fonetiche, lessicali e tematiche che contribuiscono a creare una propria metafisica, che a volte coincide e a volte no con la metafisica dantesca. La scoperta e la ricostruzione di alcune strutture base di questo linguaggio, come di uno dei tratti più caratteristici del testo della Commedia, sia in italiano che in russo, costituisce l'oggetto della mia attuale ricerca la cui tappa iniziale è sintetizzata nel presente articolo.

\section{Bibliografia}

Achmatova, Anna Andreevna. “Dante”. In: Samarina Marina Sergeevna, Šaub Igor' Jur’jevič (dir.). Dante Alig'eri: pro et contra Sankt-Peterburg: Izdatel'stvo Russkoj Christianskoj Gumanitarnoj Akademii, 2011 [1965]: 320-322.

Ariani, Marco. Lux inaccessibilis. Metafore e teologia della luce nel Paradiso di Dante. Roma: ARACNE, 2010.

Belza, Igor Fëdorovič. "Nekotorye problemy interpretacii i kommentirovanija Božestvennoj Komedii“. In: Belza Igor’ Fëdorovič, a cura di. Dantovskie Čtenija. Moskva: Nauka, 1979: 34-73. [Belza, Igor. "Alcuni problemi dell'interpretazione e del commento della "Divina Commedia”". In: Belza Igor. Letture Dantesche. Mosca: Nauka, 1979: 34-73.]

Boyde, Patrick. Perception and Passion in Dante's "Comedy. Cambridge: Cambridge University Press, 1993. [Boyde, Patrick. 'Lo color del core.' Visione, passione e ragione in Dante. Napoli: Liguori, 2002.]

Colombo, Manuela. Dai mistici a Dante: il linguaggio dell'ineffabilità. Pavia: La Nuova Italia, 1987.

Chiamenti, Massimiliano. Dante Alighieri traduttore. Firenze: Le Lettere, 1995 .

Cristiani, Maria. "Platonismo". In: Umberto Bosco, a cura di. Enciclopedia Dantesca. Roma: Istituto dell'Enciclopedia Italiana Treccani, 1977. 
Čukovskij, Kornej Ivanovič. Vysokoe iskusstvo. I principi della traduzione letteraria. [L'alta arte. I principi della traduzione letteraria]. SanktPeterburg: Avalon", 2011 [1964].

Dal', Vladimir Ivanovič. Tolkovyj slovar' živogo velikorusskago jazyka Vladimira Dalja. Tret'e, ispravlennoe i značitel'no dopolnennoe, izdanie pod redakcieju prof. I. A. Boduéna de Kurtené [Il grande dizionario di lingua russa di Vladimir Dal'. Terza edizione, rivista e notevolmente integrata, a cura del prof. Ivan Aleksandrovič Baudoin de Courtenay]. S.-PeterburgMoskva: Izdanie Tovariščestva M.O. Vol'f, 1903-1909 [1863-1866]. Messo in rete 03.03.2005. URL: http://slovari.ru/default.aspx?s=o\&p=246 (Consultato il 05. 02.2015).

Dante Alighieri. De vulgari eloquentia. A cura di Mirko Tavoni. In: Dante Alighieri. Opere. Edizione diretta da Marco Santagata. Volume primo. Milano: Mondadori, 2011.

Dante Alighieri. Epistole. In: Dante Alighieri. Tutte le opere. A cura di Luigi Blasucci. Firenze: Sansoni Editore, 1965.

Dante Alighieri. Commedia. Paradiso. Con il commento di Anna Maria Chiavacci Leonardi. Bologna: Zanichelli, 2001.

Dante Alighieri. Convivio. A cura di Giorgio Inglese. Milano: BUR, 2007 [1993].

Evgen'jeva, Anastasija Petrovna, a cura di. Slovar' russkogo jazyka $v$ 4-h tomah [Dizionario di lingua russa in quattro volumi]. Moskva: Russkij jazyk, 1981-1984 [1957-1961]. Messo in rete 03.03.2005. URL: http://slovari.ru/default.aspx? $\mathrm{s}=0 \& \mathrm{p}=240$ (Consultato il 05.02.2015).

Garbovskij, Nikolaj Konstantinovič. Teorija perevoda [La teoria della traduzione]. Moskva: Izdatel'stvo Moskovskogo universiteta, 2004.

Gasparov, Michail Leonovič. "O novom perevode "Ada”, vypolnennom V.G. Marancmanom”. In: Dante. Božestvennaja Komedija. Ad. Čistilišče. Raj. Sankt-Peterburg: Amfora, 2006: 5-8.

Gilson, Étienne. Saint Bernard [San Bernardo]. Paris: Plon, 1949.

Golenišev-Kutuzov, Il’ja Nikolaevič. "Tvorčestvo Dante i mirovaja kul'tura” [L'opera di Dante e la cultura mondiale]. In: Samarina Marina Sergeevna, Šaub Igor' Jur'jevič (dir.). Dante Alig'eri: pro et contra. Sankt-Peterburg: Izdatel'stvo Russkoj Hristianskoj Gumanitarnoj Akademii, 2011: 486-522 [1971].

Iljušin, Aleksandr Anatol'evič. "Iz nabljudenij nad stilem Božestvennoj Komedii“. In: Belza Igor' Fëdorovič, a cura di. Dantovskie Čtenija. Moskva: Nauka, 1973: 217-244. [Iljušin, Aleksandr. "Osservazioni sullo stile della Divina Commedia“. In: Belza, Igor, a cura di. Letture Dantesche. Mosca: Nauka, 1973: 217-244.]

Javelet, Robert. Image et ressemblance au douzième siècle. De Saint Anselme à Alain de Lille. Paris: Letouzey et Ané, 1967.

Lozinskij, Sergej Michajlovič. "Istorija odnogo perevoda Božestvennoj Komedii“. In: Belza Igor' Fëdorovič, a cura di. Dantovskie Čtenija. Moskva: Nauka, 1989: 10-17. [Lozinskij, Sergej. "La storia di una traduzione della Divina Commedia". In: Belza, Igor, a cura di. Letture Dantesche. Mosca: Nauka, 1989: 10-17.]

Lotman, Yurij Michajlovič. "Struktura chudožestvennogo teksta”. In: Lotman Yurij Michaijlovič. Ob iskusstve. Moskva: Iskusstvo, 1970. [Lotman, Yurij. La struttura del testo poetico, a cura di Eridano Bazzarelli. Milano: Mursia, 1972.] 
Mandel'štam, Osip Emil'jevič. "Razgovor o Dante" [1990]. In: Osip Mandel'štam. Stihotvorenija. Proza. Moskva: RIPOL-KLASSIK, 2001: 644698. [Mandel'štam, Osip. Conversazione su Dante. A cura di Remo Faccani. Genova: Il melangolo, 1994.]

Mocan, Mira. La trasparenza e il riflesso. Pavia: Bruno Mondadori Editori, 2007.

Nardi, Bruno. Saggi di filosofia dantesca. Firenze: La Nuova Italia, 1967 [1930].

Osimo, Bruno. Manuale del traduttore. Guida pratica con glossario. Milano: Hoepli, 2013 [2011].

Ožegov, Sergej Ivanovič, Švedova, Natalia Jul'evna. Tolkovyj slovar' russkogo jazyka [Dizionario di lingua russa]. Moskva: Azbukovnik, 1999 [1992]. Messo in rete il 03.03.2005. URL: http://slovari.ru/default.aspx? $\mathrm{s}=0 \& \mathrm{p}=244$. (Consultato il 05.02.2015).

Pertile, Lino. La punta del disio: semantica del desiderio nella Commedia. Fiesole (Firenze): Cadmo, 2005.

Popovič, Anton. La scienza della traduzione. Aspetti metodologici. La comunicazione traduttiva. Milano: Hoepli, 2006.

Scartazzini, Giovanni Andrea, Vandelli, Giuseppe. La Divina Commedia col commento scartazziniano rifatto da Giuseppe Vandelli, 9a ed. Milano: Hoepli, 1929. Messo in rete nel 2005. URL: http://dante.dartmouth.edu/commentaries.php (Consultato il 5 febbraio 2015).

Stabile, Giorgio. "Teoria della visione come teoria della conoscenza". In: Giorgio Stabile. Dante e la filosofia della natura. Percezioni, linguaggi, cosmologie. Roma: SISMEL Edizioni Del Galluzzo, 2007: 9-29.

Švedova, Natalia Jul'evna, a cura di. Russkij semantičeskij slovar' [Dizionario semantico russo]. Moskva: Azbukovnik, 1998. Messo in rete il 03.03.2005. URL: http://slovari.ru/default.aspx? $\mathrm{s}=\mathrm{o} \& \mathrm{p}=235$. (Consultato il 05.02.2015)

Vinogradov, Viktor Vladimirovič, a cura di. Slovar' jazyka Puškina: $v$ 4-h tomah [Dizionario della lingua di Puškin: in 4 volumi]. Moskva: Azbukovnik, 2000 [1956-1961]. Messo in rete il 03.03.2005. URL: http://slovari.ru/default.aspx?s=o\&p=230. (Consultato il 05.02.2015)

Zambon, Francesco, a cura di. Trattati d'amore cristiani del XII secolo. Volume I. Milano: Mondadori, 2007. 